next day by the improved condition of the pulse and tongue, and the rapid subsidence of the cutaneous engorgement.

I take no credit to myself for this mode of treating erysipelas, but most willingly record my obligation to Mr. Bell, of Edinburgh, who published a paper on the subject in the Monthly Journal of Medical Sciences for June, 1851. That gentleman's testimony as to the value of this treatment is most strong; he says that in every instance in his practice it has been successful. His brother, Dr. Charles Bell, is equally impressed in its favour; and he states that it not only removes the disease in a short time, but also renders the patient less susceptible of returns of the disease. In pursuing the chalybeate treatment of erysypelas, $\mathrm{Mr}$. Bell regards it as important to bring the system rapidly under its influence, and acting on this conviction, I gave in the case above related even larger doses than Mr. Bell had sanctioned.

Although the case $I$ have related was an instance of idiopathic erysipelas, the treatment is said to be equally beneficial in the traumatic form, and in infantile erysipelas. Of the latter, Mr. Bell details some remarkably interesting cases.

\section{CASE OF PUERPERAL CONVULSIONS.}

\author{
By ROBERT MARTIN, Esq., Holbrook.
}

Read before the Meeting of the Suffolk Branch, held June 2:th, $18 j 2$.

Mary Cousins, was admitted into Fattingstone Union House, at the age of seven weeks, consequent on her mother suffering from puerperal mania. The mother remains to this day a confirmed lunatic in Meiton Asylum.

Mary Cousins, the infant, was reared in the Union House, and the nurse who had charge of her states that she was frequently the subject of severe sick headache, which at times confined her to her bed for days together. She was early placed out in service, and but little is known of her, till her readmission to the Union House, in an advanced state of pregnancy. Since her readmission she is said to have suffered much from headache, but never reported herself nor authorised others to report her to the medical officer.

Mary Cousins, aged 21 years, primipara, visited by Mr. Jarmain, at 1 A.M., May 3rd, 18j2. Was in the first stage of labour, with slight pains, restless, irritable, and constantly walking about the roum; pains regular at short intervals, producing little effect on the os uteri; was suddenly seized with convulsions, on the subsidence of which she was conscious, and complained of pain in the head; skin temperate; pulse weak at the wrists, but strong at the carotids; bladder and bowels had been freely relieved.

At hali-past three A.M., I saw her, she had just recovered from a severe fit; was conscious, but rather wandering; complained of headache; pupils dilated; face but little flushed; pulse 100 ; not full, nor sharp, excepting in the carotids; pains regular at intervals of four or five minutes; os uteri dilated to nearly the size of a crown piece, thin, but rigid; membranes entire; no presentation of the child discoverable; bled to twenty ounces from the arm, with decided impression on the system; several pains succeeded without fit; the head, however, much complained of; suddenly seized with a severe fit, grinding of teeth, torticollis, opisthotonos; the tongue was protected from injury by a gag; at the termination of this fit, the head of the foetus was advanced, having descended to the hollow of the sacrum; during the fit a considerable but indefinite quantify of blond was lost from the arm into the bed, the bardage having slipped; I applied the forceps, and delivery was speedily and easily effected at half-past five, A.M.

The birth of a living child was instantly succeeded by a violent fit, with, from this moment, no recurrence of consciousness. The placenta was naturally expelled a few minutes after the fit, with more than usual loss. The uterus, however, quickly, by means of friction over its fundus and pressure, contracted firmly. Coma like a heavy sleep, supervened, without stertor or laryngismus; pulse quick and feeble. The hair was removed from the head, and cold applications employed; ten grains of calomel were placed on the back of the tongue, and the inside of the cheeks smeared with croton oil, at intervals of one hour, four times, without effect on the bowels. Mustard poultices were applied to the feet, and the region of the cervical vertebræ covered by a flannel wrung from hot water, and soaked in turpet.tine. Coina increased in intensity, laryngismus, and forcible expiration. At ten o'clock a few ounces more blood were drawn from the arm; but it was clear the system would not bear much, and death closed the scene about twelve o'clock.

Sectio-cadaveris.-Pericranium healthy; calvarium natural in density and weight, strongly adherent to the dura mater at the vertex only; dura mater natural in general appearance, but tensely stretched over the brain, especially at the posterior lobes of the cerebrum, and presenting an elevated knotty induration, of nearly an inch and a half in length, over the superior longitudinal sinus, at the posterior termination of the sagittal suture, where it had been adherent to the calvarium, and where the sinus receives the parietal veins. An incision made below this tumefaction into the superior longitudinal sinus, would not permit the fine point of my blow-pipe to be passed forwards through its track, although it was permeable by air. The induration consisted of enlarged Pacchionian glands, which springing from the pia mater, had thrust the floor and roof of the superior longitudinal sinus against the calvarium, and thus obstructing circulation through the sinus; anterior to this obstruction the sinus was much enlarged, and would readily admit $m y$ index finger; it contained several cluts of blood; on cutting round the dura mater the lobes of the brain expanded with an audible noise; the vessels of the pia mater were much distended with blood; substance of the brain healthy; horizontal sections discovered many spots of effused 
coagula, especially in the right posterior lobe; the ventricles contained no fluid; plexus choroides more pale, and less vascular than usual; base of the skull contained three ounces and a half of bloody serum, compressing the medulla oblongata at the foramen magnum.

Now, Mr. President, such a case as the one narrated, viewed irrespectively of its previous history, which can sometimes be only imperfectly, and at other times not at all arrived at, presents a grave question to the practitioners, viz.,-Do the epileptic convulsions depend solely on reflex action of the spinal nerves, or is that source of irritation complicated with intra-cranial pathology? On the first supposition, chloroform to allay spasm, with the speediest practicable delivery by turning or forceps, would be indicated; but on the second, such a case for instance as that of Mary Cousins, I fear chloroform might increase cerebral congestion, while operative measures, without its anæsthetic agency, by augmenting spinal irritation, might hasten the fatal coma, and thus rendering necessary the adoption of an ulterior proceeding. An accurate diagnosis is, therefore, of the utmost importance.

I have witnessed, I apprehend, several cases of both kinds, cerebric and excerebric, or at least I have inferred so from the results, referring to reflex action those which have recovered, leaving no trace of intra-cranial disturbance, and to cerebric origin those which have died in which my classification has been guided by postmortem inspection, but which during life presented no appreciable difference in symptoms.

Should this subject have occupied the attention of yourself, Mr. President, or of any of the members of this Association, I shall be glad to learn from you a means of clear diagnosis between puerperal epileptic convulsions dependent on reflex action of the spinal nerves simply, and those which, as in the case I have narrated, are complicated with intra-cranial pathology.

In order to obtain the opinion of that distinguished physiologist, Dr. Marshall Hall, on this subject, I addressed a letter to him, making the direct inquiry of diagnosis, and repressing the previous history of the case I sent its detail. I have now the pleasure to read you his courteous reply, from which I infer, that under any circumstances of the kind, he would not permit his patient to die without the performance of tracheotomy, and it is possible that in the case of Mary Cousins, had it been resorted to before the lesion of vessels occurred, life might have been preserved.

\section{CASE OF HAY FEVER.}

By William P. KIRKMAN, Esq., Melton.

Read before the Suffolk Branch Meeting, June 25, 1852. $\dot{-}$

Being but too personally concerned in the following case, I am induced to bring before your notice this afternoon some brief notes of a very troublesome affec- tion, which has as yet continued to bid defiance to the skill of the most eminent and learned in our profession; and when $I$ tell you that $I$ am annually afflicted with this "thorn in the flesh," and have been for the last seven years, I doubt not but that you will be kind though to give my case your favourable consideration; that I, like the bee which gathers honey from every flower, and lays it up for the ensuing year, may, from whatever suggestions you shall kindly afford, gather such an amount of therapeutical information, as will give me the chance of weilding an effectual weapon, with a view to the downfal of my annual visitant.

The disease presents many peculiarities, but little is known of its pathology ; and with respect to its cure we are in total ignorance of any remedial agent. There is one circumstance worth remarking before I proceed, which is, that very probably you will, before I have terminated my notes, (brief as they shall be,) have an ocular demonstration of some of the more urgent symptoms of a truly distressing affection, commonly known under the name of catarrhus æstivus, or hay fever. Seeking then, gentlemen, your valuable aid, I proceed to give as concisely and plainly as possible a short detail of my symptoms as they annually attack me.

Every year, at about the latter end of the month of May, a slight irritability and sensation of soreness at the inner canthi of both eyes is experienced. This continues on and off, unaccompanied by any other marked symptoms, perhaps for three or four days (no symptoms of febrile irritation whatever); to these are shortly added slight lachrymation, which subsequently becomes more profuse, and continues daily, with irritation at the inner canthi, to increase; the nostrils, one or both, become obstructed, and this is soon followed by itching, sensation of pricking and soreness in the same region; the Schneiderian membrane now begins to secrete a quantity of thin watery fluid, calling for the almost incessant use of the pocket handkerchief; in fact, by the commencement of the month of June, there are all the appearances of what is vulgarly called, " a running cold ;" these daily, but gradually, increase, and as gradually become accompanied by other concomitants, until about the end of June or the commencement of July, when the affection is at its height and presents the following distressing symptoms :-There is intense itching and irritability of the conjunctivæ, especially towards the inner canthi, as well as of the Schneiderian membrane; profuse lachrymation, and when the attacks are severe, considerable intolerance of light; violent and continned sneezing, sometimes twenty or thirty times without ceasing; the defluxion from the nostrils is immense; there is coryza and gravedo with dyspnœe, which is frequently accompanied by wheezing during inspiration; the breathing being carried on by the mouth alone, as both nostrils are blocked up by the amount of secretion given out by their lining. membrane. If these continue long, the eyes become bloodshot; there is headache, more or less severe; the pulse becomes small and weak; the tongue white and coated; shooting and darting pains about the back part of the head; lassitude and great debility. One might 